\title{
Analytic Approximation of Luminosity Distance in Cosmology via Variational Iteration Method
}

\author{
V. K. Shchigolev \\ Department of Theoretical Physics, Ulyanovsk State University, Ulyanovsk, 432000, Russia
}

Copyright (C) 2017 by authors, all rights reserved. Authors agree that this article remains permanently open access under the terms of the Creative Commons Attribution License 4.0 International License

\begin{abstract}
This work deals with a new approach in the approximate analytical representation of the luminosity distance in a homogenous Friedmann-Lemaître-RobertsonWalker (FLRW) model of the Universes by means of the variational iteration method (VIM). For the analytical calculation of the luminosity distance, we obtain the approximate solution of the differential equation which the luminosity distance obeys, using the corresponding initial conditions. On the basis of this approximate solution, a simple analytic formula for the luminosity distance as a function of redshift is obtained and compared with a numerical solution from the general integral formula.
\end{abstract}

Keywords FLRW Cosmology, Luminosity Distance, Redshift, Variational Iteration Method

\section{Introduction}

The database, obtained in the observational astronomy on Supernovae of type Ia as one of the best cosmological distance indicators [1]-[3], encourages theoretical cosmologists to a strong restriction of the essential parameters in their cosmological models. The reason is that these cosmological observations clearly prove a spatial flatness and the present acceleration of our Universe. As a result, the SNIa Union2 database [4] becomes one of the most reliable observational resources for testing various cosmological models.

In order to test any cosmological models with the help of the SNIa Union2 database, we have to use the maximumlikelihood approach minimizing $\chi^{2}$ which measures the deviations of the theoretical predictions from the observations. Since SN Ia behave as excellent standard candles, they can be used to directly measure the expansion rate of the Universe upto high redshift, comparing with the present rate. The SN Ia database gives us the distance modulus $\mu$ to each supernova. In a flat universe, the theoretical distance modulus is given by

$$
\mu(z)=5 \log _{10}\left(d_{L} / M p c\right)+25
$$

where $d_{L}$ is the luminosity distance depending on the cosmological redshift $z$ and the model parameters. Thus, the analytical calculation of the luminosity distance $d_{L}$ versus cosmological redshift $z$ seems to be a very important issue in theoretical cosmology. Unfortunately, the corresponding formula for the luminosity distance is usually expressed by an integral over the redshift, and the integration cannot be prepared explicitly. Obviously, in any case, this integration can be prepared numerically by some algorithms, but numerical methods tend to be computationally heavy when high accuracy is desired, and do not allow to carry out a reliable analytic investigation of cosmological model [5]. Therefore, an analytical approximation of the luminosity distance as a function of the redshift $z$ can be a rather useful in cosmological modeling.

For the purpose of testing different cosmologycal models, the main physical and kinematics quantities in cosmology are usually expressed in the form of a Taylor series over the redshift (see, e.g., [6]). However, the latest data on supernovae Ia can involve the redshift $z>2$, that can give rise to a theoretical question about the convergence of such a series expansion for large redshift. Therefore, one has to find some other algorithms for the analytic computing of the luminosity distance as a function of redshift. Not pretending anyway on a completeness of review, let us mention only some of these approaches.

For instance, a simple algebraic approximation for the luminosity distance in a flat universe with pressureless matter and a cosmological constant is considered in [7]. In [8], the so-called Padé approximant is applied for the analytical approximation of the luminosity distance and fitting formula. Using the elliptic integral of the first kind even in the case when all the three omega factors are non-zeros, it is derived [9] that the integral on the r.h.s. of general formula for the luminosity distance can be partly calculated analytically.

Recently, an interesting example of approximate calculation of the luminosity distance via the Homotopy Perturbation Method (HPM) [10] has been proposed in Ref. [11]. Some examples of different applications of this method in cosmology and astronomy were investigated by the author in Refs. [12]-[14]. At the same time, one more approximate 
method developed by J.-H. He, the so-called Variational Iteration Method (VIM) [15], [16], can give an approximation of high accuracy in many nonlinear problems, and can be easily implemented [17]. Indeed, this method has been successfully applied for the problems of geodesic motion in the gravitational field of astrophysical objects by the author in Ref. [18].

In this paper, we use the idea of approximate analytical calculation of the luminosity distance by virtue of solving the corresponding differential equation with certain initial conditions, proposed in [11]. Solving this equation in a spatially flat FLRW universe by means of VIM, we obtain the approximate analytical expressions for the luminosity distance in terms of redshift for the different content of cosmological models. We show that by using the VIM, the expression for $d_{L}(z)$ in arbitrary accuracy can be easily obtained by implementing a simple procedure for the governing equation.

\section{The main idea of VIM}

The VIM has been proposed by J.-H. He [15]-[17], and can be successfully applied to the linear, nonlinear, and boundary value problems. Dealing with this method, one has to construct a correction functional by a general Lagrange multiplier. Then, the Lagrange multiplier can be identified optimally using the variational theory. Being different from the other non-linear analytical methods, such as perturbation methods, this method does not depend on small parameters. It is why VIM can find wide application in non-linear problems without linearization or small perturbations. In order to demonstrate a general idea of VIM, we can consider the following general non-linear equation:

$$
L[u(x)]+N[u(x)]=g(x),
$$

where $L$ and $N$ are linear and nonlinear operators respectively, and $g(x)$ is a known function. The correct functional for the equation (1) can be given by

$u_{n+1}(x)=u_{n}(x)+\int_{0}^{x} \lambda(s)\left\{L\left[u_{n}(s)\right]+N\left[\tilde{u}_{n}(s)\right]-g(s)\right\} d s$,

where $\lambda$ is a Lagrange multiplier, that can be identified optimally using the variational iteration method. At this, $\tilde{u}_{n}$ is considered to be a restricted variation which means that $\delta \tilde{u}_{n}=0$. Making the correct functional (2) stationary, one can obtain

$$
\begin{gathered}
\delta u_{n+1}(x)=\delta u_{n}(x) \\
+\delta \int_{0}^{x} \lambda(s)\left\{L\left[u_{n}(s)\right]+N\left[\tilde{u}_{n}(s)\right]-g(s)\right\} d s \\
=\delta u_{n}(x)+\int_{0}^{x} \delta\left\{\lambda(s) L\left[u_{n}(s)\right]\right\} d s .
\end{gathered}
$$

The stationary conditions, $\delta u_{n+1}(x)=0$, can be derived using integration by parts in equation (3) and noticing that $\delta u_{n}(0)=0$. The Lagrange multipliers can be easily and precisely obtained for linear problems. However, for nonlinear problems, it is not as trivial. The nonlinear terms are treated as restricted variations such that the Lagrange multiplier can be determined as a simpler form.

The importance and the very utility of method is endowed with the choice of assumption of considering even highly nonlinear and complicated dependent variables as restricted variables thereby synchronizing the error occurring due to process of finding solution to equation (1) to its minimum magnitude. Eventually, after $\lambda$ is determined as desired, a proper selective function, may it be a linear or otherwise with respect to equation (1) is assumed as an initial approximation for finding next successive iterative function by equation (2) recursively.

The successive approximations $u_{n+1}(x)$ of the solution will be readily obtained upon using the obtained Lagrange multiplier and by using any appropriate function for $u_{0}(x)$. The zeroth approximation $u_{0}(x)$ may be selected by any function that just meets, at least, the initial and boundary conditions. Therefore by starting from $u_{0}(x)$, the exact solution may be obtained as

$$
u(x)=\lim _{n \rightarrow \infty} u_{n}(x) .
$$

Thus, in applications of VIM to differential equations, one should undertake the following three steps: (i) establishing the correction functional; (ii) identifying the Lagrange multipliers; (iii) determining the initial iteration. For the convergence criteria and error estimates of the VIM, one can refer the reader to [19]-[21].

\section{Differential Equation for the Lumi- nosity Distance}

A homogeneous isotropic universe can be described by the following FLRW metric [22],

$$
d s^{2}=-d t^{2}+a^{2}(t)\left[\frac{d r^{2}}{1-k r^{2}}+r^{2}\left(d \theta^{2}+\sin \theta d \phi^{2}\right)\right],
$$

where $a(t)$ is a scale factor, and $k=1,0,-1$ for a closed, spatially-flat, open universe respectively, and the speed of light in vacuum $c=1$. The Einstein equations,

$$
R_{i k}-\frac{1}{2} g_{i k} R+\Lambda g_{i k}=8 \pi G T_{i k},
$$

where $R_{i k}$ is the Ricci curvature tensor, $R$ is the scalar curvature, $g_{i k}$ is the metric tensor, $\Lambda$ is the cosmological constant, $G$ is Newton's gravitational constant, and $T_{i k}$ is the energy-momentum tensor of matter, leads to the first Friedmann equation in metric (5) as follows

$$
H^{2}=H_{0}^{2}\left(\sum_{m} \Omega_{m} a^{-3\left(1+w_{m}\right)}+\Omega_{k} a^{-2}+\Omega_{\Lambda}\right),
$$

where $H=\dot{a} / a$ is the Hubble parameter, $H_{0}$ denotes its present value, and we use dimensionless densities $\Omega_{m}=$ 
$\left(8 \pi G / 3 H^{2}\right) \rho_{m}, \Omega_{k}=-k / H_{0}^{2} a_{0}^{2}$ and $\Omega_{\Lambda}=\Lambda / 3 H_{0}^{2}$. Here, $\Omega_{\Lambda}$ is the contribution from the vacuum, $\Omega_{k}$ is the contribution associated with curvature, and $\Omega_{m}$ is the contribution from all other kinds of matter and fields with the equation of state parameters (EoS) $w_{m}$. As it follows from equation (6), these parameters are linearly dependent, that is

$$
\sum_{m} \Omega_{m}+\Omega_{k}+\Omega_{\Lambda}=1
$$

In the relativistic cosmology, one of the most fundamental distance scale is the luminosity distance, defined by $d_{L}=$ $\sqrt{L /(4 \pi f)}$, where $f$ is the observed flux of an astronomical object and $L$ is its luminosity. Recent astronomical observations indicate that the universe is spatially flat, and the present density parameter $\Omega_{\Lambda} \sim 0.72$. For the spatially flat universe, the luminosity distance $d_{L}$ is defined as follows

$$
d_{L}=c(1+z) \int_{o}^{z} \frac{d z^{\prime}}{H_{0} E\left(z^{\prime}\right)},
$$

where $E(z)=H(z) / H_{0}$, and $H(z)$ is the Hubble parameter (6) represented as a function of redshift $z=\left(a_{0} / a\right)-1$.

For example, the luminosity distance in the $\Lambda$ CDM model of the universe is given by [22]

$$
d_{L}(z)=\frac{c(1+z)}{H_{0}} \int_{0}^{z} \frac{d z^{\prime}}{\sqrt{\Omega_{r}\left(1+z^{\prime}\right)^{4}+\Omega_{m}\left(1+z^{\prime}\right)^{3}+\Omega_{\Lambda}}},
$$

where $\Omega_{m}, \Omega_{r}$ and $\Omega_{\Lambda}$ are the energy densities corresponding to the dust-like matter, radiation and cosmological constant, respectively, and $\Omega_{m}+\Omega_{r}+\Omega_{\Lambda}=1$ with accordance to (7). Even this rather simple example clearly shows the obvious difficulties in calculation of the integral in (9). In general, this calculation involves repeated numerical calculations, elliptic functions or some algebraic approximations.

Thus, the problem of analytical calculation of the luminosity distance remains interesting, since there exists the variety of different models in which the Hubble parameter takes rather more complicated dependence on $z$ compared to the example above. In our previous work [11], we proposed a novel approach to this problem, in which the luminosity distance can be obtained as a solution of the Cauchy problem for the differential equation followed from (8).

Let us recall this approach in brief [11]. The definition of luminosity distance (8) can be rewritten as follows

$$
d_{L}(z)=\frac{c(1+z)}{H_{0}} \int_{0}^{z} \frac{d z^{\prime}}{\sqrt{W\left(z^{\prime}\right)}}
$$

if we consider the following notation for the dimensionless Hubble parameter squared

$$
E^{2}(z)=W(z), W(z)_{\mid z=0}=1 .
$$

As a differential consequence of equation (10), we can obtain

$$
\frac{d}{d z}\left[\frac{H_{0} d_{L}}{c(1+z)}\right]=\frac{1}{\sqrt{W(z)}},
$$

and

$$
\frac{d^{2}}{d z^{2}}\left[\frac{H_{0} d_{L}}{c(1+z)}\right]=-\frac{1}{2} W^{-3 / 2}(z) \frac{d W(z)}{d z} .
$$

At last, replacing in (13) the factor $1 / \sqrt{W(z)}$ with the help of equation (12), we obtain

$$
\frac{d^{2}}{d z^{2}}\left[\frac{H_{0} d_{L}}{c(1+z)}\right]=-\frac{1}{2} \frac{d W(z)}{d z}\left(\frac{d}{d z}\left[\frac{H_{0} d_{L}}{c(1+z)}\right]\right)^{3} .
$$

In addition, equations (10)-(12) lead to the following initial conditions

$$
\left[\frac{H_{0} d_{L}}{c(1+z)}\right]_{z=0}=0, \frac{d}{d z}\left[\frac{H_{0} d_{L}}{c(1+z)}\right]_{\mid z=0}=1 .
$$

If we introduce temporally the unknown function

$$
u(z)=\frac{H_{0} d_{L}}{c(1+z)}
$$

, for the sake of simplicity, and take into account equations (14) and (15), we get the following Cauchy problem

$$
u^{\prime \prime}(z)+\frac{1}{2} W^{\prime}(z) u^{\prime 3}(z)=0 ; \quad u_{\left.\right|_{z=0}}=0, u_{\left.\right|_{z=0} ^{\prime}}=1,
$$

where the prime stands for the derivative with respect to $z$.

\section{Luminosity Distance in VIM Ap- proximation}

The main equation (17) is a nonlinear differential equation of the second order. It can be solved exactly in quadratures, but the result leads to the integral (10) once again. Therefore, we will solve the Cauchy problem (17) analytically by VIM with a certain approximation.

The comparison of equations (1) and (17) allows us to represent the correct functional (2) as follows

$$
u_{n+1}(z)=u_{n}(z)+\int_{0}^{z} \lambda_{z}(s)\left[\frac{d^{2} u_{n}(s)}{d s^{2}}+\frac{1}{2} W^{\prime}(s) \tilde{u}_{n}^{\prime 3}(s)\right] d s,
$$

where $\tilde{u}_{n}(s)$ is considered to be a restricted variation. By using the stationary condition for the correct functional (17) , we get

$$
\delta u_{n+1}(z)=\delta u_{n}(z)+\int_{0}^{z} \delta\left[\lambda_{z}(s) \frac{d^{2} u_{n}(s)}{d s^{2}}\right] d s .
$$

From stationary conditions, $\delta u_{n+1}(\varphi)=0$, and using integration by parts in Eq. (19), on can obtain the following equations for the Lagrange multiplier

$$
\left\{\begin{array}{c}
\lambda_{z}^{\prime \prime}(s)=0, \\
\lambda_{z}(s)_{\mid s=z}=0, \\
1-\lambda_{z}^{\prime}(s)_{\mid s=z}=0 .
\end{array}\right.
$$


These equations can be readily solved to obtain the following Lagrange multiplier

$$
\lambda_{z}(s)=s-z .
$$

Using this Lagrange multiplier in (18), one can readily obtain the successive approximations $u_{n}(z)$ for the solution of equation (17) getting start with some appropriate function for $u_{0}(z)$. As a result, we have the following iteration formula

$u_{n+1}(z)=u_{n}(z)+\int_{0}^{z}(s-z)\left[\frac{d^{2} u_{n}(s)}{d s^{2}}+\frac{1}{2} W^{\prime}(s) u_{n}^{\prime 3}(s)\right] d s$,

Starting with the initial approximation $u_{0}(z)=z$ in this formula, we can obtain the following results:

$$
u_{1}(z)=z+\frac{1}{2} \int_{0}^{z}(s-z) W^{\prime}(s) d s=\frac{3}{2} z-\frac{1}{2} \int_{0}^{z} W(s) d s
$$

$$
\begin{aligned}
& u_{2}(z)=\frac{3}{2} z-\frac{1}{2} \int_{0}^{z} W(s) d s \\
& -\frac{1}{2} \int_{0}^{z}(s-z)\left[1-\frac{1}{8}(3-W(s))^{3}\right] W^{\prime}(s) d s,
\end{aligned}
$$

and

$$
\begin{aligned}
& u_{3}(z)=\frac{3}{4} z+\frac{1}{64} \int_{0}^{z}(3-W(s))^{4} d s \\
& \quad-\frac{1}{16} \int_{0}^{z}(s-z)(3-W(s))^{3} W^{\prime}(s) d s \\
& +\frac{1}{2} \int_{0}^{z}(s-z)\left[\frac{3}{4}+\frac{1}{64}(3-W(s))^{4}\right]^{3} W^{\prime}(s) d s \\
& =z+\frac{1}{2} \int_{0}^{z}(s-z)\left[\frac{3}{4}+\frac{1}{64}(3-W(s))^{4}\right]^{3} W^{\prime}(s) d s,
\end{aligned}
$$

which satisfy the initial conditions (17) due to the normalization condition $W(0)=1$, that is equation (11). Then taking into account (16), and (26) - (28), we can express the corresponding approximations for the luminosity distance as follows

$$
d_{L}^{(1)}(z)=\frac{c(1+z)}{H_{0}}\left[\frac{3}{2} z-\frac{1}{2} \int_{0}^{z} W(s) d s\right],
$$

for the first-order approximation,

$$
d_{L}^{(2)}(z)=\frac{c(1+z)}{H_{0}}\left[\frac{3}{4} z+\frac{1}{64} \int_{0}^{z}(3-W(s))^{4} d s\right],
$$

for the second-order approximation, and

$$
\begin{gathered}
d_{L}^{(3)}(z)=\frac{c(1+z)}{H_{0}}\left[\frac{31}{65} z+\right. \\
+\frac{1}{128} \int_{0}^{z}\left(27+\frac{27}{80}(3-W(s))^{4}+\frac{1}{256}(3-W(s))^{8}\right. \\
\left.\left.+\frac{1}{13 \cdot 4096}(3-W(s))^{12}\right)(3-W(s)) d s\right]
\end{gathered}
$$

for the third-order approximation. It should be noted that the approximate formulas (26)-(28) can be easily applied not only to the models with a polynomial dependence in $(1+z)$ of the Hubble parameter squared, as it is in the $\Lambda$ CDM model, but also to many other FLWR models where the computation of the integral in (8) becomes problematic at all. The proposed approach allows to get an analytic expression for the function $d_{L}(z)$, at least by using the Maple package.

\section{Application Examples}

First of all, let us consider a simple example of the luminosity distance in the $\Lambda \mathrm{CDM}$ model of the universe given by (9) where $\Omega_{r}+\Omega_{m}+\Omega_{\Lambda}=1$. Observations show that the radiation density is very small today, $\Omega_{r} \sim 10^{-4}$, and this term can be neglected. Therefore, we use now

$$
W(z)=\Omega_{m}(1+z)^{3}+\Omega_{\Lambda},
$$

and $\Omega_{\Lambda}=1-\Omega_{m}$. Substituting (29) in (26) and (27), we obtain the following approximations for the luminosity distance in this model:

$$
\begin{array}{r}
d_{L}^{(1)}(z)=\frac{c}{H_{0}} z\left[1+\left(1-\frac{3}{4} \Omega_{m}\right) z-\frac{5}{4} \Omega_{m} z^{2}\right. \\
\left.-\frac{5}{8} \Omega_{m} z^{3}-\frac{1}{2} \Omega_{m} z^{4}\right],
\end{array}
$$

and

$$
\begin{aligned}
& d_{L}^{(2)}(z)= \frac{c}{H_{0}}(z+1)\left[\frac{3}{4} z+\frac{1}{832} M^{4}\left((z+1)^{13}-1\right)\right. \\
&-\frac{1}{160}(2+M) M^{3}\left((z+1)^{10}-1\right) \\
&+\frac{3}{224}(2+M)^{2} M^{2}\left((z+1)^{7}-1\right) \\
&\left.-\frac{1}{64}(2+M)^{3} M\left((z+1)^{4}-1\right)+\frac{1}{64}(2+M)^{4} z\right]
\end{aligned}
$$

Substituting (29) into equation (25), one can easily obtain the luminosity distance in the third-order approximation. We do not provide it here because of its cumbersome nature.

We can compare these approximations with well known expansion of the luminosity distance $d_{L}$ in a Taylor series in redshift $z$, that is (see, e.g., [23])

$d_{L}(z)=\frac{c z}{H_{0}}\left[1+\frac{1}{2}\left(1-q_{0}\right) z-\frac{1}{6}\left(1-q_{0}-3 q_{0}^{2}+j_{0}\right) z^{2}+O\left(z^{3}\right)\right]$, 


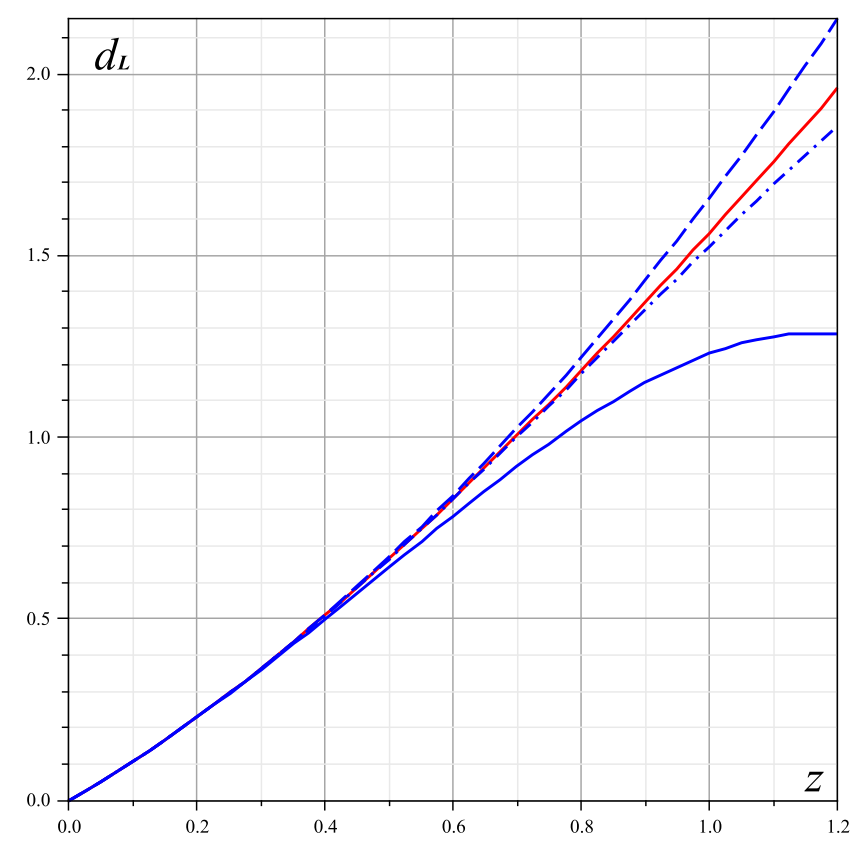

Figure 1. Comparison of the numerical solution to Eq. (9) for the $\Lambda$ CDM model (red line) with the approximate solutions given by (26) (blue solid line), (27) (blue dashed line), and (28) (blue dot-dashed line).

where the present magnitudes of the deceleration parameter $q=-a \ddot{a} / \dot{a}^{2}$ and the jerk parameter $j=a^{2} \ddot{a} / \dot{a}^{3}$ are denoted as $q_{0}$ and $j_{0}$, respectively. By using (32), both equations (30) and (31) lead to the same expression for $q_{0}$ as follows

$$
q_{0}=-1+\frac{3}{2} \Omega_{m}
$$

In the framework of this paper, we are not trying to constrain parameters of this model with the observational data. For the illustrative purpose only, let us insert $\Omega_{\Lambda}=0.72$ and $\Omega_{m}=0.28$ in (29). Then we have $q_{0} \approx-0.58$, which is within the observational limits.

Table 1. Percentage of relative errors of the corresponding approximations to $d_{L}$ given by Eqs. (26), (27) and (28).

\begin{tabular}{|c||l|l|l|}
\hline$z$ & $\%$ by $d_{L}^{(1)}$ & \multicolumn{1}{|c|}{$\%$ by $d_{L}^{(2)}$} & $\%$ by $d_{L}^{(3)}$ \\
\hline \hline 0.1 & 0.0989 & 0.00326 & 0.0001 \\
\hline 0.2 & 0.4409 & 0.0301 & 0.0017 \\
\hline 0.3 & 1.098 & 0.1153 & 0.0099 \\
\hline 0.4 & 2.1457 & 0.3043 & 0.0363 \\
\hline 0.5 & 3.6642 & 0.6497 & 0.1004 \\
\hline 0.6 & 5.7351 & 1.2042 & 0.2318 \\
\hline 0.7 & 8.4419 & 2.0118 & 0.4708 \\
\hline 0.8 & 11.8697 & 3.0972 & 0.8678 \\
\hline 0.9 & 16.1048 & 4.4579 & 1.4812 \\
\hline 1.0 & 21.2349 & 6.0624 & 2.3729 \\
\hline 1.1 & 27.3491 & 7.8615 & 3.6029 \\
\hline 1.2 & 34.5383 & 9.8250 & 5.2277 \\
\hline
\end{tabular}

With the help of Maple package, the graphs of $d_{L}(z)$ in units of $c / H_{0}$ for the numerical solutions to the integral in equation (9), and the approximate solutions (26) - (28) are shown in Fig. 1, where we have used $\Omega_{m}=0.28, \Omega_{\Lambda}=$
$0.72, \Omega_{r}=0$. Besides, Table 1 shows the percentage of relative errors of the approximate solutions compared to the numerical one.

For the next example, we consider a spatially flat universe with the quintessential matter. Let the equation of state parameter be equals to $-1<w_{q}<-1 / 3$. Then equation (6) becomes as follows

$$
W(z)=\Omega_{m}(1+z)^{3}+\Omega_{q}(1+z)^{3\left(1+w_{q}\right)},
$$

which, being substituted in (26), yields

$$
\begin{aligned}
d_{L}^{(1)}(z)= & \frac{c(1+z)}{H_{0}}\left[\frac{3}{2} z-\frac{\Omega_{m}}{8}\left((1+z)^{4}-1\right)\right. \\
& \left.-\frac{1-\Omega_{m}}{2\left(4+3 w_{q}\right)}\left((1+z)^{4+3 w_{q}}-1\right)\right] .
\end{aligned}
$$

Just as easy, the explicit expressions for $d_{L}^{(2)}(z)$ and $d_{L}^{(3)}(z)$ can be obtained from equations (27), (28) and (34). The graphs of all approximations in this case are similar to those in Fig. 1, from which we could conclude that the first-order approximation may be useful only for $z \ll 1$. This is the obvious consequence of coincidence of (10) and (26) followed from the decomposition

$$
\frac{1}{\sqrt{W\left(z^{\prime}\right)}}=\frac{1}{\sqrt{1+\left(W\left(z^{\prime}\right)-1\right)}} \approx 1-\frac{1}{2}\left[W\left(z^{\prime}\right)-1\right],
$$

which can provide an acceptable accuracy only for $z \ll 1$. Nevertheless, even the first-order approximation could be improved by some additional tuning. Let us consider an example of such a possibility.

Suppose that we want to improve the accuracy of the firstorder approximation (26) in a vicinity of the point $z=z_{0}$. For this end, we can insert an indefinite parameter $p_{1}$ in equation (23), say, as follows

$$
u_{1}(z)=\frac{3}{2} p z-\frac{1}{2}(1-p) \int_{0}^{z} W(s) d s .
$$

At the same time, the exact expression for $u(z)$ is given by (10) as $u(z)=\int_{0}^{z} \frac{d s}{\sqrt{W(s)}}$. The necessary condition for the minimum of difference between $u(z)$ and $u_{1}(z)$ from (23), that is $\left[u(z)-u_{1}(z)\right]_{\mid z=z_{0}}^{\prime}=0$, yields

$$
p=\frac{2+W\left(z_{0}\right) \sqrt{W\left(z_{0}\right)}}{\left[3+W\left(z_{0}\right)\right] \sqrt{W\left(z_{0}\right)}} .
$$

As an example, let us put $z_{0}=1, \Omega_{m}=0.28$ and $w_{q}=$ $-2 / 3$ in equations (29) and (34). One can obtain from (37) that $p \approx 0.7$ in both cases with different accuracy. Therefore, we have

$$
\begin{array}{r}
d_{L}^{(\Lambda)}(z)=\frac{c(1+z)}{H_{0}}\left[\frac{3}{2} p z-(1-p) \frac{\Omega_{m}}{8}\left((1+z)^{4}-1\right)\right. \\
\left.+(1-p) \frac{1-\Omega_{m}}{2} z\right]
\end{array}
$$


for the $\Lambda$ CDM model, and

$$
\begin{aligned}
d_{L}^{(q)}(z)=\frac{c(1+z)}{H_{0}} & {\left[\frac{3}{2} p z-(1-p) \frac{\Omega_{m}}{8}\left((1+z)^{4}-1\right)\right.} \\
& \left.-(1-p) \frac{1-\Omega_{m}}{4}\left((1+z)^{2}-1\right)\right]
\end{aligned}
$$

for the qCDM model with $w_{q}=-2 / 3$.

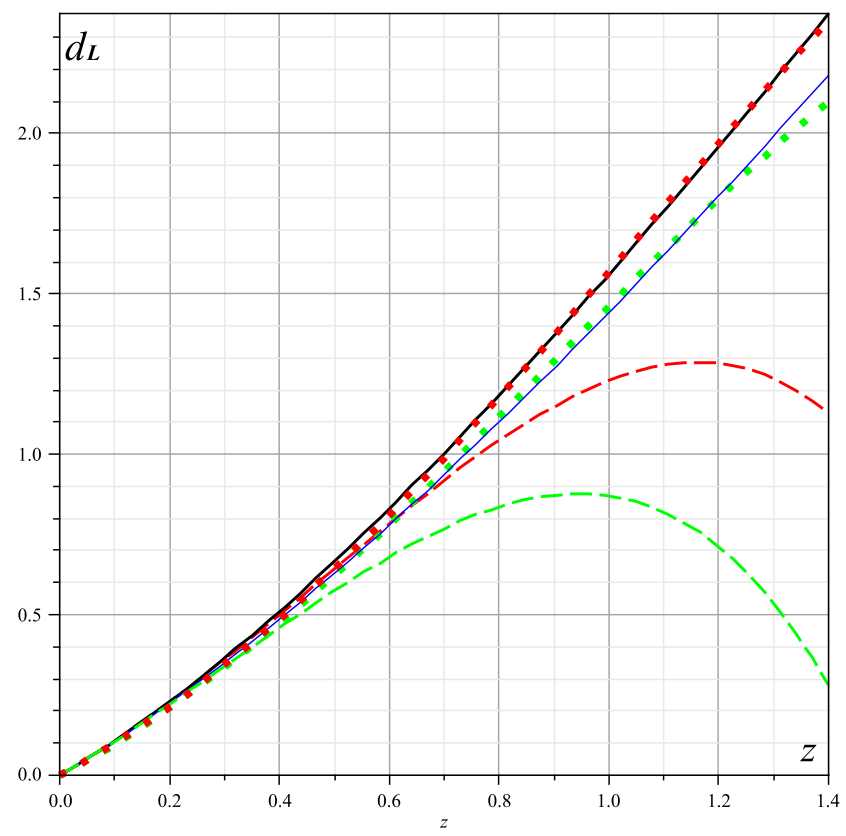

Figure 2. Comparison of the numerical solution to Eq. (9) for the $\Lambda \mathrm{CDM}$ model (black line) and qCDM model (blue line) with the approximate solutions given by (38) (red dot line) and (39) (green dot line), and the corresponding first-order approximations, (30) and (35) (dashed lines).

Table 2. Percentage of relative errors of the corrected approximations to $d_{L}$ given by Eqs. (38) and (39).

\begin{tabular}{|c||l|l|}
\hline$z$ & \multicolumn{1}{|c|}{$\%$ by $d_{L}^{(\Lambda)}$} & \multicolumn{1}{|c|}{$\%$ by $d_{L}^{(q)}$} \\
\hline \hline 0.70 & 1.4096 & 0.85718 \\
\hline 0.75 & 0.9688 & 1.13112 \\
\hline 0.80 & 0.5731 & 1.32456 \\
\hline 0.85 & 0.2269 & 1.43455 \\
\hline 0.90 & 0.0656 & 1.45812 \\
\hline 0.95 & 0.3004 & 1.39219 \\
\hline 1.00 & 0.4736 & 1.23365 \\
\hline 1.05 & 0.5809 & 0.97930 \\
\hline 1.10 & 0.6186 & 0.62589 \\
\hline 1.15 & 0.5826 & 0.17009 \\
\hline 1.20 & 0.4688 & 0.39144 \\
\hline 1.25 & 0.2733 & 1.06216 \\
\hline 1.30 & 0.0078 & 1.84559 \\
\hline 1.35 & 0.3785 & 2.74519 \\
\hline
\end{tabular}

Fig. 2 shows the corresponding numerical solutions of (9) for the $\Lambda \mathrm{CDM}$ model (black solid line) and the qCDM model (blue solid line) compared to the corresponding approximations (red and green lines, respectively). The dot lines represent the improved approximations given by equations
(38) and (39). The dashed lines represent the corresponding first-order approximations by equations (30) and (35).

The obvious advantage of the corrected approximations given by (38) and (39) becomes even more clear from Table 2, where we represent the percentage of relative errors provided by these equations. It can be readily concluded that the relative errors of the simple approximations by (38) and (39) are mostly less then $1 \%$ for the redshift within the vicinity of 1 . It can be assumed that an even more accurate approximations can be obtained by a similar method applied to the secondand third-order approximations.

\section{Conclusions}

Thus, a simple analytical computation of the luminosity distance in relativistic cosmology via the Variational Iteration Method has been considered. For this end, we have used the proposed earlier by the author conversion of the problem of calculating the integral in the well-known expression for the luminosity distance (8) to the problem of solving the Cauchy problem of the corresponding nonlinear differential equation (17). In this paper, the approximate solution of this equation is performed by using VIM in a direct way without using any restrictive assumption. Therefore, the computation becomes simple but rather effective, at least, in a vicinity of some value of $z$. The relative accuracy of this method is briefly analyzed from the graphical representations of approximations obtained, and the numerical evaluation of their relative errors. It would be noted that this method can be extended to cover the variety of cosmological models with different expressions for $W(z)$. This approximation seems to be useful in the models involving $W(z)$ in the form of non-polynomial over $(1+z)$ expression.

\section{REFERENCES}

[1] A. G. Riess, et al. Observational Evidence from Supernovae for an Accelerating Universe and a Cosmological Constant, Astronomical Journal, Vol. 116, 1009, 1998. http://dx.doi.org/10.1086/300499

[2] S. Perlmutter, et al. Measurements of Omega and Lambda from 42 High-Redshift Supernovae, Astrophysical Journal, Vol. 517, 565, 1999. http://dx.doi.org/10.1086/307221

[3] N. Suzuki, D. Rubin, C. Lidman, et al. The Hubble Space Telescope Cluster Supernova Survey. V. Improving The DarkEnergy Constraints Above $z>1$ and Building an Early-TypeHosted Supernova Sample, Astrophysical Journal, 746, 85, 2012.

[4] R. Amanullah, et al. Spectra and Light Curves of Six Type Ia Supernovae at $0.511<z<1.12$ and the Union2 Compilation, Astrophysical Journal, Vol. 716, 712-738, 2010. http://dx.doi.org/10.1088/0004-637X/716/1/712

[5] De-Zi Liu, Cong Ma, Tong-Jie Zhang, and Zhiliang Yang. Numerical Strategies of Computing the Luminosity Dis- 
tance, Mon. Not. R. Astron. Soc., Vol. 412, 2685, 2011. http://dx.doi.org/10.1111/j.1365-2966.2010.18101.x

[6] Céline Cattoën and Matt Visser. The Hubble series: convergence properties and redshift variables, Class. Quantum Grav., Vol. 24, 5985, 2007. http://dx.doi.org/10.1088/0264$9381 / 24 / 23 / 018$

[7] Ue-Li Pen. Analytical Fit to the Luminosity Distance for Flat Cosmologies with a Cosmological Constant, Astrophys. J. Suppl. S., Vol. 120, 4950, 1999.

[8] Hao Wei, Xiao-Peng Yan, Ya-Nan Zhou. Cosmological Applications of Pade Approximant, JCAP, Vol. 1401, 045, 2014. http://dx.doi.org/10.1088/1475-7516/2014/01/045

[9] A. Meszaros, J. Ripa. On the relation between the non-flat cosmological models and the elliptic integral of first kind, Astron. Astrophys., Vol. 573, A54, 2015. https://doi.org/10.1051/0004-6361/201425201

[10] J.-H. He. Homotopy perturbation technique, Computer Methods in Applied Mechanics and Engineering, Vol. 178, 257262, 1999. http://dx.doi.org/10.1016/S0045-7825(99)000183

[11] V. K. Shchigolev. Calculating Luminosity Distance versus Redshift in FLRW Cosmology via Homotopy Perturbation Method, arXiv:1511.07459.

[12] V. Shchigolev. Homotopy Perturbation Method for Solving a Spatially Flat FRW Cosmological Model, Universal Journal of Applied Mathematics, Vol.2, No. 2, 99-103, 2014. http://dx.doi.org/10.13189/ujam.2014.020204

[13] V. Shchigolev. Analytical Computation of the Perihelion Precession in General Relativity via the Homotopy Perturbation Method, Universal Journal of Computational Mathematics, Vol. 3, No. 4, 45-49, 2015. http://dx.doi.org/10.13189/ujcmj.2015.030401

[14] V. K. Shchigolev, D. N. Bezbatko. On HPM approximation for the perihelion preces-sion angle in general relativity, International Journal of Advanced Astronomy, Vol. 5, No. 1, 38-43, 2017. http://dx.doi.org/10.14419/ijaa.v5i1.7279.
[15] J.-H. He. Variational iteration method - a kind of non-linear analytical technique: some examples, International Journal of Non-Linear Mechanics, Vol. 34, No. 4, 699-708, 1999. http://dx.doi.org/10.1016/S0020-7462(98)00048-1

[16] J.-H. He. Variational iteration method for autonomous ordinary differential systems, Applied Mathematics and Computation, Vol. 114, No. 2-3, 115-123, 2000. http://dx.doi.org/10.1016/S0096-3003(99)00104-6

[17] J.-H. He. Variational iteration method-Some recent results and new interpretations, Journal of Computational and Applied Mathematics, Vol. 207, No. 1, 3-17, 2007. http://dx.doi.org/10.1016/j.cam.2006.07.009

[18] V. K. Shchigolev. Variational iteration method for studying perihelion precession and deflection of light in General Relativity, International Journal of Physical Research, Vol. 4, No. 2, 52-57, 2016. http://dx.doi.org/10.14419/ijpr.v4i2.6530

[19] M. Tatari and M. Dehghan. On the Convergence of He's Variational Iteration Method, Journal of Computational and Applied Mathematics, Vol. 207, No. 1, 121-128, 2007. http://dx.doi.org/10.1016/j.cam.2006.07.017

[20] J. I. Ramos. On the Variational Iteration Method and Other Iterative Techniques for Nonlinear Differential Equations, $A p$ plied Mathematics and Computation, Vol. 199, No. 1, 39-69, 2008. http://dx.doi.org/10.1016/j.amc.2007.09.024

[21] Ernest Scheiber. On the Convergence of the Variational Iteration Method, arxiv:1509.01779.

[22] S. Weinberg, Gravitation and Cosmology: Principles and Applications of The General Theory of Relativity (John Wiley. Press, New York, 1972).

[23] Takeshi Chiba, Takashi Nakamura. The Luminosity Distance, the Equation of State, and the Geometry of the Universe, Prog. Theor. Phys., Vol.100, 1077, 1998. http://dx.doi.org/10.1143/PTP.100.1077 